\title{
COMPLETE CHARACTERISATION OF KRONECKER INVARIANTS OF A MATRIX PENCIL WITH A PRESCRIBED QUASI-REGULAR SUBPENCIL*
}

\author{
MARIJA DODIG ${ }^{\dagger}$
}

\begin{abstract}
In this paper, the possible Kronecker invariants of a matrix pencil with a prescribed quasi-regular subpencil are determined.
\end{abstract}

Key words. Completion of matrix pencils, Quasi-regular pencils, Polynomial chains.

AMS subject classifications. 15A83, 05A17.

1. Introduction. Matrix and matrix pencils completion problems have been thoroughly studied in the past. The General Matrix Pencil Completion Problem (GMPCP) consists of determining the possible Kronecker invariants of a matrix pencil with a prescribed subpencil. For the motivation and most important classical results, see e.g. [1,2,4,21,24,25,27,29-32]. There are also new insights to matrix pencils completion problems coming from Representation Theory (see $[22,28]$ ), but still far away from the compact and explicit form of solutions. However, these problem have been put away from the main stream of Modern Linear Algebra, despite its connection to Control Theory and Linear Systems (both Singular and Regular), and possible applications in engineering, see e.g. [3,7,20,23,26]. In the last decade, we have done significant effort in bringing these problems back to light. Many new combinatorial structures and results were obtained while trying to find the best path toward solution of GMPCP, see [5, 8-10,13-16, 19]. The results obtained in $[8,11,12]$ have made possible to obtain a solution to many important cases of GMPCP.

Overall, there are two different approaches in studying GMPCP. The minimal one is a novel approach that deals with GMPCP by restricting the dimension of the completion. By following this approach, we have used up all its force and solved minimal case completion of GMPCP in [8]. The other, classical approach consists in restricting the number of invariants, i.e., by restricting the structure of the involved matrix pencils. In this paper, we follow the classical approach and come close to GMPCP as possible, by studying and solving the following problem:

Problem 1. Characterise the possible Kronecker invariants of a matrix pencil with a prescribed quasiregular subpencil.

In Theorem 3.1, we give a complete, explicit and constructive solution to Problem 1. In this way, we generalize results in $[1,2,4,6,9,10,13,16,22,24,27-31]$. Indeed, for example in [9] and [13] we have solved Problem 1 with additional restrictions on the resulting pencil - in [9], we have required that the resulting pencil is without nontrivial homogeneous invariant factors, and in [13], we have considered it to be quasi-

${ }^{*}$ Received by the editors on February 10, 2020. Accepted for publication on May 7, 2020. Handling Editor: Froilán M. Dopico.

${ }^{\dagger}$ CEAFEL, Departamento de Matématica, Universidade de Lisboa, Edificio C6, Campo Grande, 1749-016 Lisbon, Portugal (msdodig@fc.ul.pt), and Mathematical Institute SANU, Knez Mihajlova 36, 11000 Beograd, Serbia. This work was done within the activities of CEAFEL and was partially supported by FCT, projects UIDB/04721/2020 and IF/01232/2014/CP1216/CT0012, and by the Ministry of Science, Technology and Development of Republic of Serbia, project no. 174020 . 
regular. In [10], we have studied and solved the minimal case of Problem 1, with restrictions on the size of a completion. Also, in [6] and [16] Problem 1 with additional assumption on the prescribed quasi-regular subpencil was solved - in [16], we assumed that it was without nontrivial homogeneous invariant factors, while in [6], it was assumed to be regular. In this paper, by using powerful recent result on polynomial chains from [12], we give a solution to Problem 1 in full generality. This solution naturally generalizes [6,9,10,13,16] as well as many related classical results like [1,2,4,24,27, 29-31].

The paper is organised in four sections and one appendix. In Section 2, we give definitions and auxiliary results that will be used in the proof of the main result. In Section 3, we write Problem 1 as a combinatorial problem, and we give the main result of the paper (Theorem 3.1). In Section 4, we prove the main result. Also, there is an Appendix in which we prove that partitions $\mathbf{f}$ and $\mathbf{w}$, appearing in the main result, are well defined.

2. Auxiliary results. Let $\mathbb{F}$ be an algebraically closed field. All polynomials throughout the paper are assumed to be homogeneous polynomials from $\mathbb{F}[\lambda, \mu]$, and monic with respect to $\lambda$. For details on homogeneous polynomials see e.g. [5]. Let $\alpha_{1}|\cdots| \alpha_{n}$ be any chain of homogeneous polynomials, we assume $\alpha_{i}=1$, for all $i<1$ and $\alpha_{i}=0$, for all $i>n$. By $d\left(\alpha_{i}\right)$ we denote the degree of a polynomial $\alpha_{i}$.

By a partition we mean a non increasing sequence of integers. For any integers $a_{1} \geq \cdots \geq a_{s}$ we define a corresponding partition by $\mathbf{a}=\left(a_{1}, \ldots, a_{s}\right)$. Moreover, for any such sequence, we assume $a_{i}=+\infty$, for $i \leq 0$, and $a_{i}=-\infty$, for $i>s$. Also, for any integers $c_{1}, \ldots, c_{r}$, we put $\sum_{i=a}^{b} c_{i}=0$ whenever $a>b$.

In [16], we have introduced the concept of generalized majorization:

Definition 2.1. Let $d_{1} \geq \cdots \geq d_{m+k-s}, g_{1} \geq \cdots \geq g_{m+k}, a_{1} \geq \cdots \geq a_{s}$ be integers. Consider partitions $\mathbf{d}=\left(d_{1}, \ldots, d_{m+k-s}\right), \mathbf{g}=\left(g_{1}, \ldots, g_{m+k}\right)$ and $\mathbf{a}=\left(a_{1}, \ldots, a_{s}\right)$. If

$$
\begin{gathered}
d_{i} \geq g_{i+s}, \quad i=1, \ldots, m+k-s, \\
\sum_{i=1}^{h_{j}} g_{i}-\sum_{i=1}^{h_{j}-j} d_{i} \leq \sum_{i=1}^{j} a_{i}, \quad j=1, \ldots, s \\
\sum_{i=1}^{m+k} g_{i}=\sum_{i=1}^{m+k-s} d_{i}+\sum_{i=1}^{s} a_{i},
\end{gathered}
$$

where

$$
h_{j}:=\min \left\{i \mid d_{i-j+1}<g_{i}\right\}, \quad j=1, \ldots, s,
$$

then we say that $\mathbf{g}$ is majorized by $\mathbf{d}$ and $\mathbf{a}$. This type of majorization we call the generalized majorization, and we write

$$
\mathrm{g} \prec^{\prime}(\mathbf{d}, \mathbf{a}) \text {. }
$$

Notice that, if (2.3) is satisfied, then (2.2) is equivalent to the following:

$$
\sum_{i=h_{j}+1}^{m+k} g_{i} \geq \sum_{i=h_{j}-j+1}^{m+k-s} d_{i}+\sum_{i=j+1}^{s} a_{i}, \quad j=1, \ldots, s .
$$

Definition 2.2. If partitions $\mathbf{a}, \mathbf{d}$ and $\mathbf{g}$ in Definition 2.1 satisfy (2.1), (2.4) and

$$
\sum_{i=1}^{m+k} g_{i} \geq \sum_{i=1}^{m+k-s} d_{i}+\sum_{i=1}^{s} a_{i}
$$


then we say that $\mathbf{g}$ is weakly majorized by $\mathbf{d}$ and $\mathbf{a}$, and we write

$$
\mathbf{g} \prec^{\prime \prime}(\mathbf{d}, \mathbf{a}) \text {. }
$$

REMARK 2.3. We note that (2.4) for $j=s$, and (2.1) together imply:

$$
\sum_{i=h_{s}+1}^{m+k} g_{i} \geq \sum_{i=h_{s}-s+1}^{m+k-s} d_{i} \geq \sum_{i=h_{s}-s+1}^{m+k-s} g_{i+s},
$$

i.e.,

$$
d_{i}=g_{i+s}, \quad \text { for all } i=h_{s}-s+1, \ldots, m+k-s .
$$

Let $h_{0}:=0$, then (2.2) is trivially satisfied for $j=0$. In addition, if (2.5) is valid, then (2.4) is also trivially satisfied for $j=0$.

Let $\mathbf{c}=\left(c_{1}, \ldots, c_{m}\right)$ and $\mathbf{d}=\left(d_{1}, \ldots, d_{m+x-s}\right)$ be two partitions of nonnegative integers, and let $\mathbf{a}=\left(a_{1}, \ldots, a_{s}\right)$ and $\mathbf{b}=\left(b_{1}, \ldots, b_{x}\right)$ be partitions of integers.

Let $\bar{\Omega}(\mathbf{c}, \mathbf{d}, \mathbf{a}, \mathbf{b})$ be the following condition: there exists a partition $\mathbf{g}=\left(g_{1}, \ldots, g_{m+x}\right)$ of nonnegative integers such that

$$
\mathbf{g} \prec^{\prime \prime}(\mathbf{d}, \mathbf{a}) \quad \text { and } \quad \mathbf{g} \prec^{\prime \prime}(\mathbf{c}, \mathbf{b}) \text {. }
$$

We shall use this condition in our main result.

In [13], we obtained the following result:

ThEOREM 2.4. Let $A(\lambda) \in \mathbb{F}[\lambda]^{n \times(n+m)}$ be a quasi-regular matrix pencil. Denote by $\alpha_{1}|\cdots| \alpha_{n}$ and $c_{1} \geq$ $\cdots \geq c_{c}>c_{c+1}=\cdots=c_{m}=0$ its homogeneous invariant factors and column minimal indices, respectively. Let $Q(\lambda) \in \mathbb{F}[\lambda]^{(n+s) \times(n+m+x)}$ be a quasi-regular matrix pencil, $s \leq m+x$. Denote by $\beta_{1}|\cdots| \beta_{n+s}$ and $d_{1} \geq \cdots \geq d_{d}>d_{d+1}=\cdots=d_{m+x-s}=0$ its homogeneous invariant factors and column minimal indices, respectively.

There exist matrix pencils $X(\lambda) \in \mathbb{F}[\lambda]^{n \times x}, Y(\lambda) \in \mathbb{F}[\lambda]^{s \times(n+m)}$, and $Z(\lambda) \in \mathbb{F}[\lambda]^{s \times x}$, such that the pencil

$$
\left[\begin{array}{c|c}
A(\lambda) & X(\lambda) \\
\hline Y(\lambda) & Z(\lambda)
\end{array}\right]
$$

is strictly equivalent to $Q(\lambda)$ if and only if

$$
\begin{aligned}
& c \leq s+d, \\
& \beta_{i}\left|\alpha_{i}\right| \beta_{i+x+s}, \quad i=1, \ldots, n,
\end{aligned}
$$

and there exists a partition of nonnegative integers $\mathbf{g}=\left(g_{1}, \ldots, g_{m+x}\right)$ satisfying:

$$
\begin{aligned}
& \mathrm{g} \prec^{\prime}(\mathbf{c}, \mathbf{b}), \\
& \mathrm{g} \prec^{\prime}(\mathbf{d}, \mathbf{a}),
\end{aligned}
$$

where $\mathbf{a}=\left(a_{1}, \ldots, a_{s}\right)$ and $\mathbf{b}=\left(b_{1}, \ldots, b_{x}\right)$ are given by

$$
a_{j}=d\left(\Psi_{s-j+1}\right)-d\left(\Psi_{s-j}\right)-1, \quad j=1, \ldots, s,
$$




$$
b_{j}=d\left(\Phi_{j-1}\right)-d\left(\Phi_{j}\right), \quad j=1, \ldots, x,
$$

with $\Psi_{j}=\prod_{i=1}^{n+j} \operatorname{lcm}\left(\psi_{i-j}, \beta_{i}\right), j=0, \ldots, s$, and $\Phi_{j}=\prod_{i=1}^{n} \operatorname{lcm}\left(\psi_{i}, \alpha_{i-j}\right), j=0, \ldots, x$, where

$$
\psi_{i}:=\operatorname{gcd}\left(\alpha_{i}, \beta_{i+s}\right), \quad i=1, \ldots, n \text {. }
$$

Also, as a direct corollary of [18, Lemma 2.4], we have the following:

Theorem 2.5. Let $\mathbf{c}=\left(c_{1}, \ldots, c_{m}\right), \mathbf{d}=\left(d_{1}, \ldots, d_{m+x-s}\right), \mathbf{a}=\left(a_{1}, \ldots, a_{s}\right)$, and $\mathbf{b}=\left(b_{1}, \ldots, b_{x}\right)$, be partitions such that

$$
\sum_{i=1}^{m+x-s} d_{i}+\sum_{i=1}^{s} a_{i}=\sum_{i=1}^{m} c_{i}+\sum_{i=1}^{x} b_{i} .
$$

If there exists a partition $\mathbf{g}^{\prime}=\left(g_{1}^{\prime}, \ldots, g_{m+x}^{\prime}\right)$ such that

$$
\mathbf{g}^{\prime} \prec^{\prime \prime}(\mathbf{d}, \mathbf{a}) \quad \text { and } \quad \mathbf{g}^{\prime} \prec^{\prime \prime}(\mathbf{c}, \mathbf{b})
$$

then there exists a partition $\mathbf{g}=\left(g_{1}, \ldots, g_{m+x}\right)$ such that

$$
\mathbf{g} \prec^{\prime}(\mathbf{d}, \mathbf{a}) \quad \text { and } \quad \mathrm{g} \prec^{\prime}(\mathbf{c}, \mathbf{b}) \text {. }
$$

Moreover, if $\mathbf{g}^{\prime}$ is a partition of nonnegative integers and $\sum_{i=1}^{m+x-s} d_{i}+\sum_{i=1}^{s} a_{i}+\geq 0$, then there exists a partition $\mathbf{g}$ of nonnegative integers satisfying (2.11).

In [12], we have studied polynomial chains. We cite here a version of Theorem 1 from [12] as will be used in the proof of the main result:

THEOREM 2.6. Let $x, y$ and $s$ be nonnegative integers such that $y \geq s$. Let $\alpha: \alpha_{1}|\cdots| \alpha_{n}$ and $\gamma:$ $\gamma_{1}|\cdots| \gamma_{n+s}$ be polynomial chains. Let $X_{1}, \ldots, X_{x+s-1}, Y_{1}, \ldots, Y_{y-s-1}$ and $Z$ be nonnegative integers.

There exists a polynomial chain $\beta: \beta_{1}|\cdots| \beta_{n+s}$ satisfying

$$
\begin{gathered}
\beta_{i}\left|\alpha_{i}\right| \beta_{i+x+s}, \quad i=1, \ldots, n, \\
\gamma_{i}\left|\beta_{i}\right| \gamma_{i+y-s}, \quad i=1, \ldots, n+s, \\
\sum_{i=1}^{n+s} d\left(\beta_{i}\right)=Z, \\
\sum_{i=1}^{n+\min (x+s-j, s)} d\left(\operatorname{lcm}\left(\alpha_{i-x-s+j}, \beta_{i}\right)\right) \leq X_{j}, \quad j=1, \ldots, x+s-1, \\
\sum_{i=1}^{n+s} d\left(\operatorname{lcm}\left(\beta_{i-k}, \gamma_{i}\right)\right) \leq Y_{k}, \quad k=1, \ldots, y-s-1
\end{gathered}
$$

if and only if

$$
\begin{gathered}
\gamma_{i}\left|\alpha_{i}\right| \gamma_{i+x+y}, \quad i=1, \ldots, n, \\
\sum_{i=1}^{n+\min (s, x+s-j+k)} d\left(\operatorname{lcm}\left(\alpha_{i-x-s+j-k}, \gamma_{i}\right)\right) \leq X_{j}+Y_{k}-Z,
\end{gathered}
$$

for $j=0, \ldots, x+s, k=0, \ldots, y-s$, such that $j \leq x$ or $k=0$.

Here $X_{x+s}=\sum_{i=1}^{n} d\left(\alpha_{i}\right), X_{0}=Z, Y_{y-s}=\sum_{i=1}^{n+s} d\left(\gamma_{i}\right)$ and $Y_{0}=Z$. 
Also, we shall use the following result from [19, Lemma 10]:

Lemma 2.7. Let $A(\lambda) \in \mathbb{F}[\lambda]^{(n+p) \times(n+m+l)}$ and $B(\lambda) \in \mathbb{F}[\lambda]^{l \times(n+m+l)}$ be matrix pencils, $n=\operatorname{rank} A(\lambda)$. Let $M(\lambda)=\left[\begin{array}{c}A(\lambda) \\ B(\lambda)\end{array}\right]$ and let $s=\operatorname{rank} M(\lambda)-\operatorname{rank} A(\lambda)$. There exist matrix pencils $X(\lambda) \in \mathbb{F}[\lambda]^{s \times(n+m+l)}$ and $Y(\lambda) \in \mathbb{F}[\lambda]^{(l-s) \times(n+m+l)}$ such that $\left[\begin{array}{c}A(\lambda) \\ X(\lambda) \\ Y(\lambda)\end{array}\right]$ is strictly equivalent to $M(\lambda)$, and such that $\left[\begin{array}{c}A(\lambda) \\ X(\lambda)\end{array}\right]$ has rank equal to $n+s$, and has the same row minimal indices as $A(\lambda)$, and the same column minimal indices as $M(\lambda)$.

3. Main result. Let $A(\lambda) \in \mathbb{F}[\lambda]^{n \times(n+m)}$ be a quasi-regular matrix pencil, hence $\operatorname{rank} A(\lambda)=n$. Denote by $\alpha_{1}|\cdots| \alpha_{n}$ and $c_{1} \geq \cdots \geq c_{m}$ its homogeneous invariant factors and column minimal indices, respectively. Let us denote by $c$ the number of nonzero column minimal indices of $A(\lambda)$.

Let $M(\lambda) \in \mathbb{F}[\lambda]^{(n+y) \times(n+m+x)}$ be a matrix pencil, with $\operatorname{rank} M(\lambda)=n+s$. Denote by $\gamma_{1}|\cdots| \gamma_{n+s}$, $d_{1} \geq \cdots \geq d_{m+x-s}$ and $\bar{r}_{1} \geq \cdots \geq \bar{r}_{y-s}$ its homogeneous invariant factors, column and row minimal indices, respectively. Let us denote by $d$ the number of nonzero column minimal indices of $M(\lambda)$.

By the ranks of the pencils $A(\lambda)$ and $M(\lambda)$, we have

$$
\sum_{i=1}^{n} d\left(\alpha_{i}\right)+\sum_{i=1}^{m} c_{i}+s=\sum_{i=1}^{n+s} d\left(\gamma_{i}\right)+\sum_{i=1}^{m+x-s} d_{i}+\sum_{i=1}^{y-s} \bar{r}_{i}
$$

Let us define integers $f_{1}, \ldots, f_{s}$ by

$$
\sum_{i=j+1}^{s} f_{i}=\sum_{i=1}^{n+s-j} d\left(\operatorname{lcm}\left(\alpha_{i-s+j}, \gamma_{i}\right)\right)-\sum_{i=1}^{n} d\left(\alpha_{i}\right)-s+j, \quad j=0, \ldots, s-1,
$$

and integers $w_{1}, \ldots, w_{x}$ by

$$
\sum_{i=j+1}^{x} w_{i}=\max _{k=0, \ldots, y-s}\left[\sum_{i=1}^{n+s} d\left(\operatorname{lcm}\left(\alpha_{i-s-j-k}, \gamma_{i}\right)\right)-\sum_{i=k+1}^{y-s} \bar{r}_{i}-\sum_{i=1}^{n+s} d\left(\gamma_{i}\right)\right], \quad j=0, \ldots, x-1 .
$$

In Appendix, we prove that if $\gamma_{i} \mid \alpha_{i}, i=1, \ldots, n$, then $f_{1} \geq \cdots \geq f_{s} \geq-1$. Also, we prove that if the condition

$$
\sum_{i=1}^{n+s} d\left(\operatorname{lcm}\left(\alpha_{i-s-x-k}, \gamma_{i}\right)\right) \leq \sum_{i=k+1}^{y-s} \bar{r}_{i}+\sum_{i=1}^{n+s} d\left(\gamma_{i}\right), \quad k=0, \ldots, y-s
$$

is valid, then $w_{1} \geq \cdots \geq w_{x} \geq 0$. Thus, if $\gamma_{i} \mid \alpha_{i}, i=1, \ldots, n$, and (3.22) holds we have that both partitions $\mathbf{f}=\left(f_{1}, \ldots, f_{s}\right)$ and $\mathbf{w}=\left(w_{1}, \ldots, w_{x}\right)$ are well defined

Now we can give the main result:

TheOREM 3.1. Let $A(\lambda) \in \mathbb{F}[\lambda]^{n \times(n+m)}$ be a quasi-regular matrix pencil and $M(\lambda) \in \mathbb{F}[\lambda]^{(n+y) \times(n+m+x)}$ be a matrix pencil, as above. There exist matrix pencils $X(\lambda) \in \mathbb{F}[\lambda]^{n \times x}, Y(\lambda) \in \mathbb{F}[\lambda]^{y \times(n+m)}$, and $Z(\lambda) \in$ $\mathbb{F}[\lambda]^{y \times x}$, such that the pencil

$$
\left[\begin{array}{c|c}
A(\lambda) & X(\lambda) \\
\hline Y(\lambda) & Z(\lambda)
\end{array}\right]
$$


is strictly equivalent to $M(\lambda)$ if and only if

$$
\begin{array}{ll}
\text { (i) } & c \leq d+s, \\
\text { (ii) } & \gamma_{i}\left|\alpha_{i}\right| \gamma_{i+x+y}, \quad i=1, \ldots, n, \\
\text { (iii) } & \sum_{i=1}^{n+s} d\left(\operatorname{lcm}\left(\alpha_{i-s-x-k}, \gamma_{i}\right)\right) \leq \sum_{i=k+1}^{y-s} \bar{r}_{i}+\sum_{i=1}^{n+s} d\left(\gamma_{i}\right), \quad k=0, \ldots, y-s, \\
\text { (iv) } & \bar{\Omega}(\mathbf{c}, \mathbf{d}, \mathbf{f}, \mathbf{w}) \text { holds. }
\end{array}
$$

REMARK 3.2. Once [11] is published, the condition $\bar{\Omega}(\mathbf{c}, \mathbf{d}, \mathbf{f}, \mathbf{w})$ in Theorem 3.1 can be replaced with its explicit form $\Omega(\mathbf{c}, \mathbf{d}, \mathbf{f}, \mathbf{w})$ given in [11].

3.1. Properties of the pencil (3.23) and its subpencils. Since $A(\lambda)$ is a quasi-regular matrix pencil, so is the pencil

$$
[A(\lambda) \mid X(\lambda)] \in \mathbb{F}[\lambda]^{n \times(n+m+x)}
$$

and its rank equals $n$. Moreover, since the rank of (3.23) is $n+s$, by Lemma 2.7 we have that the pencil (3.23) is strictly equivalent to the pencil

$$
\left[\begin{array}{c|c}
A(\lambda) & X(\lambda) \\
Y_{1}(\lambda) & Z_{1}(\lambda) \\
\hline Y_{2}(\lambda) & Z_{2}(\lambda)
\end{array}\right],
$$

where the subpencil

$$
\left[\begin{array}{cc}
A(\lambda) & X(\lambda) \\
Y_{1}(\lambda) & Z_{1}(\lambda)
\end{array}\right] \in \mathbb{F}[\lambda]^{(n+s) \times(n+m+x)}
$$

is such that its rank is exactly $n+s$, i.e., it is quasi-regular and its column minimal indices coincide with the column minimal indices of $(3.23)\left(d_{1} \geq \cdots \geq d_{m+x-s}\right)$.

Let us denote the homogeneous invariant factors of (3.25) by $\beta_{1}|\cdots| \beta_{n+s}$.

Next, let us consider the completion of $A(\lambda)$ up to (3.25). It is a completion from a quasi-regular up to a quasi-regular matrix pencil, so we can apply Theorem 2.4:

A completion from $A(\lambda)$ up to (3.25) is possible if and only if there exists a partition of nonnegative integers $\mathbf{g}=\left(g_{1}, \ldots, g_{m+x}\right)$ satisfying conditions (2.7)-(2.10). We note that from the ranks of the involved matrix pencils we also have

$$
\sum_{i=1}^{n} d\left(\alpha_{i}\right)+\sum_{i=1}^{m} c_{i}+s=\sum_{i=1}^{n+s} d\left(\beta_{i}\right)+\sum_{i=1}^{m+x-s} d_{i}
$$

By the definition of the general majorization, (2.9) is equivalent to

$$
\begin{gathered}
c_{i} \geq g_{i+x}, \quad i=1, \ldots, m, \\
\sum_{i=h_{j}+1}^{m+x} g_{i}-\sum_{i=h_{j}-j+1}^{m} c_{i} \geq \sum_{i=j+1}^{x} b_{i}, \quad j=0, \ldots, x,
\end{gathered}
$$


and

$$
\sum_{i=1}^{m+x} g_{i}=\sum_{i=1}^{m} c_{i}+\sum_{i=1}^{x} b_{i}
$$

where $h_{j}:=\min \left\{i \mid c_{i-j+1}<g_{i}\right\}, j=1, \ldots, x$, and $h_{0}:=0$.

By the definition of $b_{i}$ 's, if (2.8) is satisfied, then we have that

$$
\sum_{i=j+1}^{x} b_{i}=\sum_{i=1}^{n+s} d\left(\operatorname{lcm}\left(\alpha_{i-j-s}, \beta_{i}\right)\right)-\sum_{i=1}^{n+s} d\left(\beta_{i}\right), \quad j=0, \ldots, x-1 .
$$

Hence, (3.28) becomes

$$
\sum_{i=1}^{n+s} d\left(\operatorname{lcm}\left(\alpha_{i-j-s}, \beta_{i}\right)\right) \leq \sum_{i=h_{j}+1}^{m+x} g_{i}-\sum_{i=h_{j}-j+1}^{m} c_{i}+\sum_{i=1}^{n+s} d\left(\beta_{i}\right)
$$

for all $j=0, \ldots, x$. Also, (3.29) becomes

$$
\sum_{i=1}^{m+x} g_{i}=\sum_{i=1}^{m} c_{i}+\sum_{i=1}^{n+s} d\left(\operatorname{lcm}\left(\alpha_{i-s}, \beta_{i}\right)\right)-\sum_{i=1}^{n+s} d\left(\beta_{i}\right) .
$$

Analogously, by the definition of the general majorization, (2.10) is equivalent to

$$
\begin{gathered}
d_{i} \geq g_{i+s}, \quad i=1, \ldots, m+x-s, \\
\sum_{i=v_{j}+1}^{m+x} g_{i}-\sum_{i=v_{j}-j+1}^{m+x-s} d_{i} \geq \sum_{i=j+1}^{s} a_{i}, \quad j=0, \ldots, s,
\end{gathered}
$$

and

$$
\sum_{i=1}^{m+x} g_{i}=\sum_{i=1}^{m+x-s} d_{i}+\sum_{i=1}^{s} a_{i}
$$

where $v_{j}:=\min \left\{i \mid d_{i-j+1}<g_{i}\right\}, j=1, \ldots, s$, and $v_{0}:=0$.

By the definition of $a_{i}$ 's, if (2.8) is satisfied, then we have that

$$
\sum_{i=j+1}^{s} a_{i}=\sum_{i=1}^{n+s-j} d\left(\operatorname{lcm}\left(\alpha_{i-s+j}, \beta_{i}\right)\right)-\sum_{i=1}^{n} d\left(\alpha_{i}\right)-s+j, \quad j=0, \ldots, s-1
$$

Hence, (3.33) becomes

$$
\sum_{i=1}^{n+s-j} d\left(\operatorname{lcm}\left(\alpha_{i-s+j}, \beta_{i}\right)\right) \leq \sum_{i=v_{j}+1}^{m+x} g_{i}-\sum_{i=v_{j}-j+1}^{m+x-s} d_{i}+\sum_{i=1}^{n} d\left(\alpha_{i}\right)+s-j, \quad j=0, \ldots, s
$$


and (3.34) becomes

$$
\sum_{i=1}^{m+x} g_{i}=\sum_{i=1}^{m+x-s} d_{i}+\sum_{i=1}^{n+s} d\left(\operatorname{lcm}\left(\alpha_{i-s}, \beta_{i}\right)\right)-\sum_{i=1}^{n} d\left(\alpha_{i}\right)-s .
$$

We note that if (3.26) is valid, then the righthand sides of (3.31) and (3.36) coincide, as needed.

Finally, let us consider the row completion from (3.25) up to (3.23). By Theorem 2 in [15] (see also $[14,19])$, we have that this completion is possible if and only if the following conditions are valid:

$$
\begin{aligned}
& \gamma_{i}\left|\beta_{i}\right| \gamma_{i+y-s}, \quad i=1, \ldots, n+s, \\
& \sum_{i=1}^{n+s} d\left(\beta_{i}\right)=\sum_{i=1}^{n+s} d\left(\gamma_{i}\right)+\sum_{i=1}^{y-s} \bar{r}_{i}, \\
& \sum_{i=1}^{k} \bar{r}_{i} \leq \sum_{i=1}^{k} \hat{b}_{i}, \quad k=1, \ldots, y-s
\end{aligned}
$$

where

$$
\sum_{i=1}^{k} \hat{b}_{i}=\sum_{i=1}^{y-s} \bar{r}_{i}+\sum_{i=1}^{n+s} d\left(\gamma_{i}\right)-\sum_{i=1}^{n+s} d\left(\operatorname{lcm}\left(\beta_{i-k}, \gamma_{i}\right)\right), \quad k=1, \ldots, y-s .
$$

Hence, (3.39) is equivalent to

$$
\sum_{i=1}^{n+s} d\left(\operatorname{lcm}\left(\beta_{i-k}, \gamma_{i}\right)\right) \leq \sum_{i=k+1}^{y-s} \bar{r}_{i}+\sum_{i=1}^{n+s} d\left(\gamma_{i}\right), \quad k=1, \ldots, y-s .
$$

REMARK 3.3. We note that if (3.37) and (3.38) hold, the inequality (3.40) is also trivially satisfied for $k=0$.

Finally, as the result of the above analysis we conclude that our starting completion problem given in Theorem 3.1 is equivalent to the existence of homogeneous polynomials $\beta_{1}|\cdots| \beta_{n+s}$ and a partition $\mathbf{g}=\left(g_{1}, \ldots, g_{m+x}\right)$ of nonnegative integers, which satisfy conditions (2.7), (2.8), (3.26), (3.27), (3.30), (3.31), (3.32), (3.35), (3.36), (3.37), (3.38) and (3.40).

Now we pass to the proof of Theorem 3.1 given in the following section.

\section{Proof of Theorem 3.1.}

4.1. Necessity of the conditions $(i)-(i v)$. Let us suppose that there exists the wanted completion. As we have shown in Section 3.1 that is equivalent to the existence of homogeneous polynomials $\beta_{1}|\cdots| \beta_{n+s}$ and a partition $\mathbf{g}=\left(g_{1}, \ldots, g_{m+x}\right)$ of nonnegative integers, which satisfy conditions (2.7), (2.8), (3.26), (3.27), (3.30), (3.31), (3.32), (3.35), (3.36), (3.37), (3.38) and (3.40).

Condition (2.7) is equal to condition (i). Conditions (2.8) and (3.37) give (ii). Conditions (2.8), (3.38) and (3.40) give (iii).

Before proceeding with the proof of $(i v)$, we shall write conditions (3.30), (3.35) and (3.40) in slightly different equivalent forms, according to the notation from Theorem 2.6. Let

$$
Z:=\sum_{i=1}^{y-s} \bar{r}_{i}+\sum_{i=1}^{n+s} d\left(\gamma_{i}\right)
$$


Consider condition (3.30). By (3.38), it is equal to

$$
\sum_{i=1}^{n+s} d\left(\operatorname{lcm}\left(\alpha_{i-x+j-s}, \beta_{i}\right)\right) \leq \sum_{i=h_{x-j}+1}^{m+x} g_{i}-\sum_{i=h_{x-j}-x+j+1}^{m} c_{i}+Z, \quad j=0, \ldots, x .
$$

Let us denote by

$$
X_{j}:=\sum_{i=h_{x-j}+1}^{m+x} g_{i}-\sum_{i=h_{x-j}-x+j+1}^{m} c_{i}+Z, \quad j=0, \ldots, x
$$

Then (4.42) becomes

$$
\sum_{i=1}^{n+s} d\left(\operatorname{lcm}\left(\alpha_{i-x-s+j}, \beta_{i}\right)\right) \leq X_{j}, \quad j=0, \ldots, x
$$

Next consider condition (3.35). It is equal to

$$
\sum_{i=1}^{n+x+s-j} d\left(\operatorname{lcm}\left(\alpha_{i-x-s+j}, \beta_{i}\right)\right) \leq \sum_{i=v_{j-x}+1}^{m+x} g_{i}-\sum_{i=v_{j-x}-j+x+1}^{m+x-s} d_{i}+\sum_{i=1}^{n} d\left(\alpha_{i}\right)+s-j+x
$$

for all $j=x, \ldots, x+s$. Let us denote by

$$
X_{j}:=\sum_{i=v_{j-x}+1}^{m+x} g_{i}-\sum_{i=v_{j-x}-j+x+1}^{m+x-s} d_{i}+\sum_{i=1}^{n} d\left(\alpha_{i}\right)+s-j+x, \quad j=x, \ldots, x+s .
$$

Now (4.45) becomes

$$
\sum_{i=1}^{n+x+s-j} d\left(\operatorname{lcm}\left(\alpha_{i-x-s+j}, \beta_{i}\right)\right) \leq X_{j}, \quad j=x, \ldots, x+s .
$$

Note that for $j=x$ the definitions of $X_{x}$ from (4.43) and (4.46) coincide. Also, we note that $X_{0}=Z$, and $X_{x+s}=\sum_{i=1}^{n} d\left(\alpha_{i}\right)$.

Finally, let us denote by

$$
Y_{k}:=\sum_{i=k+1}^{y-s} \bar{r}_{i}+\sum_{i=1}^{n+s} d\left(\gamma_{i}\right), \quad k=0, \ldots, y-s
$$

By Remark 3.3, (3.40) becomes

$$
\sum_{i=1}^{n+s} d\left(\operatorname{lcm}\left(\beta_{i-k}, \gamma_{i}\right)\right) \leq Y_{k}, \quad k=0, \ldots, y-s
$$

Note that $Y_{0}=Z$, and $Y_{y-s}=\sum_{i=1}^{n+s} d\left(\gamma_{i}\right)$. 
Hence, (4.44), (4.47) and (4.49), together with (2.8), (3.37), (3.38) and (4.41), by Theorem 2.6 give that the following is valid:

$$
\begin{gathered}
\gamma_{i}\left|\alpha_{i}\right| \gamma_{i+x+y}, \quad i=1, \ldots, n, \\
\sum_{i=1}^{n+\min (s, x+s-j+k)} d\left(\operatorname{lcm}\left(\alpha_{i-x-s+j-k}, \gamma_{i}\right)\right) \leq X_{j}+Y_{k}-Z,
\end{gathered}
$$

for $j=0, \ldots, x+s, k=0, \ldots, y-s$, such that $j \leq x$ or $k=0$.

Due to restriction on the indices $j$ and $k(j \leq x$ or $k=0)$, condition (4.51) can be split into

$$
\sum_{i=1}^{n+x+s-j} d\left(\operatorname{lcm}\left(\alpha_{i-x-s+j}, \gamma_{i}\right)\right) \leq X_{j}, \quad j=x, \ldots, x+s,
$$

and

$$
\sum_{i=1}^{n+s} d\left(\operatorname{lcm}\left(\alpha_{i-x-s+j-k}, \gamma_{i}\right)\right) \leq X_{j}+Y_{k}-Z, \quad j=0, \ldots, x, \quad k=0, \ldots, y-s .
$$

Before proceeding we note that (4.52) for $j=x$ coincide with (4.53) for $j=x$ and $k=0$.

From the definition of $X_{j}, j=x, \ldots, x+s$, condition (4.52) is equal to

$$
\sum_{i=v_{j-x}+1}^{m+x} g_{i}-\sum_{i=v_{j-x}-j+x+1}^{m+x-s} d_{i} \geq \sum_{i=1}^{n+x+s-j} d\left(\operatorname{lcm}\left(\alpha_{i-x-s+j}, \gamma_{i}\right)\right)-\sum_{i=1}^{n} d\left(\alpha_{i}\right)-s+j-x
$$

for $j=x, \ldots, x+s$. The last is equal to

$$
\sum_{i=v_{j}+1}^{m+x} g_{i}-\sum_{i=v_{j}-j+1}^{m+x-s} d_{i} \geq \sum_{i=1}^{n+s-j} d\left(\operatorname{lcm}\left(\alpha_{i-s+j}, \gamma_{i}\right)\right)-\sum_{i=1}^{n} d\left(\alpha_{i}\right)-s+j, \quad j=0, \ldots, s .
$$

Since the condition $(\mathrm{ii})$ is valid, from the definition of the weak generalized majorization, and by the definition (3.20) of $\mathbf{f}=\left(f_{1}, \ldots, f_{s}\right)$, we have that (3.32) and (4.55) are equivalent to

$$
\mathbf{g} \prec^{\prime \prime}(\mathbf{d}, \mathbf{f}) \text {. }
$$

As for condition (4.53), by the definition of $X_{j}, j=0, \ldots, x$ and $Y_{k}, k=0, \ldots, y-s$, it is equal to

$$
\sum_{i=h_{x-j}+1}^{m+x} g_{i}-\sum_{i=h_{x-j}-x+j+1}^{m} c_{i} \geq \sum_{i=1}^{n+s} d\left(\operatorname{lcm}\left(\alpha_{i-x-s+j-k}, \gamma_{i}\right)\right)-\sum_{i=k+1}^{y-s} \bar{r}_{i}-\sum_{i=1}^{n+s} d\left(\gamma_{i}\right)
$$

for all $j=0, \ldots, x, k=0, \ldots, y-s$.

The last is equal to

$$
\sum_{i=h_{j}+1}^{m+x} g_{i}-\sum_{i=h_{j}-j+1}^{m} c_{i} \geq \max _{k=0, \ldots, y-s}\left[\sum_{i=1}^{n+s} d\left(\operatorname{lcm}\left(\alpha_{i-s-j-k}, \gamma_{i}\right)\right)-\sum_{i=k+1}^{y-s} \bar{r}_{i}-\sum_{i=1}^{n+s} d\left(\gamma_{i}\right)\right]
$$


for all $j=0, \ldots, x$.

Since the condition $(\mathrm{iii})$ is valid, from the definition of the weak generalized majorization, and by the definition (3.21) of $\mathbf{w}=\left(w_{1}, \ldots, w_{x}\right)$, we have that (3.27) and (4.58) are equivalent to

$$
\mathrm{g} \prec^{\prime \prime}(\mathbf{c}, \mathbf{w}) .
$$

Finally, (4.56) and (4.59) give (iv) as wanted.

4.2. Sufficiency of the conditions $(i)-(i v)$. Let us suppose that conditions $(i)-(i v)$ are valid. We are left with proving the existence of homogeneous polynomials $\beta_{1}|\cdots| \beta_{n+s}$ and a partition of nonnegative integers $\mathbf{g}=\left(g_{1}, \ldots, g_{m+x}\right)$ which satisfy conditions (2.7), (2.8), (3.26), (3.27), (3.30), (3.31), (3.32), (3.35), (3.36), (3.37), (3.38) and (3.40).

First note that $(i)$ coincide with (2.7). Next, we have that condition (iv) implies the existence of a partition $\mathbf{g}^{\prime}=\left(g_{1}, \ldots, g_{m+x}\right)$ of nonnegative integers such that

$$
\mathbf{g}^{\prime} \prec^{\prime \prime}(\mathbf{c}, \mathbf{w})
$$

and

$$
\mathbf{g}^{\prime} \prec^{\prime \prime}(\mathbf{d}, \mathbf{f})
$$

By the definition of the weak generalized majorization, together with the definition of $\mathbf{w}$ from (3.21), we have that (4.60) is equivalent to

$$
c_{i} \geq g_{i+x}^{\prime}, \quad i=1, \ldots, m
$$

and

$$
\sum_{i=h_{j}^{\prime}+1}^{m+x} g_{i}^{\prime}-\sum_{i=h_{j}^{\prime}-j+1}^{m} c_{i} \geq \sum_{i=1}^{n+s} d\left(\operatorname{lcm}\left(\alpha_{i-s-j-k}, \gamma_{i}\right)\right)-\sum_{i=k+1}^{y-s} \bar{r}_{i}-\sum_{i=1}^{n+s} d\left(\gamma_{i}\right)
$$

for all $j=0, \ldots, x, k=0, \ldots, y-s$, where $h_{j}^{\prime}:=\min \left\{i \mid c_{i-j+1}<g_{j}^{\prime}\right\}, j=1, \ldots, x, h_{0}^{\prime}:=0$.

Also, by the definition of the weak generalized majorization, together with the definition of $\mathbf{f}$ from (3.20), condition (4.61) is equivalent to

$$
d_{i} \geq g_{i+s}^{\prime}, \quad i=1, \ldots, m+x-s
$$

and

$$
\sum_{i=v_{j}^{\prime}+1}^{m+x} g_{i}^{\prime}-\sum_{i=v_{j}^{\prime}-j+1}^{m+x-s} d_{i} \geq \sum_{i=1}^{n+s-j} d\left(\operatorname{lcm}\left(\alpha_{i-s+j}, \gamma_{i}\right)\right)-\sum_{i=1}^{n} d\left(\alpha_{i}\right)-s+j, \quad j=0, \ldots, s,
$$

where $v_{j}^{\prime}:=\min \left\{i \mid d_{i-j+1}<g_{j}^{\prime}\right\}, j=1, \ldots, s, v_{0}^{\prime}:=0$.

Equation (4.63) is equal to

$$
\sum_{i=h_{x-j}^{\prime}+1}^{m+x} g_{i}^{\prime}-\sum_{i=h_{x-j}^{\prime}-x+j+1}^{m} c_{i} \geq \sum_{i=1}^{n+s} d\left(\operatorname{lcm}\left(\alpha_{i-x-s+j-k}, \gamma_{i}\right)\right)-\sum_{i=k+1}^{y-s} \bar{r}_{i}-\sum_{i=1}^{n+s} d\left(\gamma_{i}\right)
$$


Electronic Journal of Linear Algebra, ISSN 1081-3810

A publication of the International Linear Algebra Society

Volume 36, pp. 430-445, July 2020.

for all $j=0, \ldots, x, k=0, \ldots, y-s$.

Equation (4.65) is equal to

$$
\sum_{i=v_{j-x}^{\prime}+1}^{m+x} g_{i}^{\prime}-\sum_{i=v_{j-x}^{\prime}-j+x+1}^{m+x-s} d_{i} \geq \sum_{i=1}^{n+s-j+x} d\left(\operatorname{lcm}\left(\alpha_{i-s+j-x}, \gamma_{i}\right)\right)-\sum_{i=1}^{n} d\left(\alpha_{i}\right)-s+j-x
$$

for all $j=x, \ldots, x+s$.

Let $Y_{k}$ be as in (4.48), and let $Z$ be as in (4.41).

Let

$$
\begin{gathered}
X_{j}^{\prime}:=\sum_{i=h_{x-j}^{\prime}+1}^{m+x} g_{i}^{\prime}-\sum_{i=h_{x-j}^{\prime}-x+j+1}^{m} c_{i}+Z, \quad j=0, \ldots, x, \\
X_{j}^{\prime}:=\sum_{i=v_{j-x}^{\prime}+1}^{m+x} g_{i}^{\prime}-\sum_{i=v_{j-x}^{\prime}-j+x+1}^{m+x-s} d_{i}+\sum_{i=1}^{n} d\left(\alpha_{i}\right)+s-j+x, \quad j=x, \ldots, x+s .
\end{gathered}
$$

We note that $X_{0}^{\prime}\left(=Y_{0}\right)=Z$, and $X_{s+x}^{\prime}=\sum_{i=1}^{n} d\left(\alpha_{i}\right)$.

Then we write (4.66) and (4.67) together as

$$
\begin{gathered}
\sum_{i=1}^{n+\min (s, x+s-j+k)} d\left(\operatorname{lcm}\left(\alpha_{i-x-s+j-k}, \gamma_{i}\right)\right) \leq X_{j}^{\prime}+Y_{k}-Z, \\
\text { for } j=0, \ldots, x+s, \quad k=0, \ldots, y-s, \text { such that } j \leq x \text { or } k=0 .
\end{gathered}
$$

By Theorem 2.6, conditions (ii) and (4.70) give the existence of homogeneous polynomials $\beta_{1}|\cdots| \beta_{n+s}$ which satisfy

$$
\sum_{i=1}^{n+s} d\left(\beta_{i}\right)=Z
$$

conditions $(2.8),(3.37),(3.40)$,

$$
\sum_{i=1}^{n+s} d\left(\operatorname{lcm}\left(\alpha_{i-s-j}, \beta_{i}\right)\right) \leq \sum_{i=h_{j}^{\prime}+1}^{m+x} g_{i}^{\prime}-\sum_{i=h_{j}^{\prime}-j+1}^{m} c_{i}+\sum_{i=1}^{n+s} d\left(\beta_{i}\right), \quad j=0, \ldots, x
$$

and

$$
\sum_{i=1}^{n+s-j} d\left(\operatorname{lcm}\left(\alpha_{i-s+j}, \beta_{i}\right)\right) \leq \sum_{i=v_{j}^{\prime}+1}^{m+x} g_{i}^{\prime}-\sum_{i=v_{j}^{\prime}-j+1}^{m+x-s} d_{i}+\sum_{i=1}^{n} d\left(\alpha_{i}\right)+s-j, \quad j=0, \ldots, s-1
$$

We note that (4.73) is also trivially satisfied for $j=s$.

Condition (4.71) is equal to (3.38), and (4.71) together with (3.19) gives (3.26). 
If denote by

$$
\sum_{i=j+1}^{x} b_{i}^{\prime}:=\sum_{i=1}^{n+s} d\left(\operatorname{lcm}\left(\alpha_{i-s-j}, \beta_{i}\right)\right)-\sum_{i=1}^{n+s} d\left(\beta_{i}\right), \quad j=0, \ldots, x-1,
$$

and by

$$
\sum_{i=j+1}^{s} a_{i}^{\prime}:=\sum_{i=1}^{n+s-j} d\left(\operatorname{lcm}\left(\alpha_{i-s+j}, \beta_{i}\right)\right)-\sum_{i=1}^{n} d\left(\alpha_{i}\right)-s+j, \quad j=0, \ldots, s-1,
$$

conditions (4.62) and (4.72), as well as (4.64) and (4.73), give

$$
\mathbf{g}^{\prime} \prec^{\prime \prime}\left(\mathbf{c}, \mathbf{b}^{\prime}\right) \quad \text { and } \quad \mathbf{g}^{\prime} \prec^{\prime \prime}\left(\mathbf{d}, \mathbf{a}^{\prime}\right) \text {. }
$$

Here $\mathbf{a}^{\prime}=\left(a_{1}^{\prime}, \ldots, a_{s}^{\prime}\right)$ and $\mathbf{b}^{\prime}=\left(b_{1}^{\prime}, \ldots, b_{x}^{\prime}\right)$.

By (3.26), we have $\sum_{i=1}^{m} c_{i}+\sum_{i=1}^{x} b_{i}^{\prime}=\sum_{i=1}^{m+x-s} d_{i}+\sum_{i=1}^{s} a_{i}^{\prime} \geq 0$. By Theorem 2.5, we have that (4.76) implies the existence of a partition $\mathbf{g}=\left(g_{1}, \ldots, g_{m+x}\right)$ of nonnegative integers such that

$$
\mathrm{g} \prec^{\prime}\left(\mathbf{c}, \mathbf{b}^{\prime}\right) \quad \text { and } \quad \mathrm{g} \prec^{\prime}\left(\mathbf{d}, \mathbf{a}^{\prime}\right) \text {. }
$$

By the definition of the generalized majorization condition (4.77) give conditions (3.27), (3.30), (3.31), (3.32), (3.35) and (3.36), as wanted.

This finishes our proof.

Appendix. The following lemma is a simple generalization of [17, Lemma 2]:

Lemma 4.1. Let $\bar{\alpha}: \bar{\alpha}_{1}|\cdots| \bar{\alpha}_{n}$ and $\bar{\gamma}: \bar{\gamma}_{1}|\cdots| \bar{\gamma}_{m}$ be two chains of monic polynomials. Let

$$
\pi_{j}(\bar{\alpha}, \bar{\gamma})=\prod_{i=\min (1, j+1)}^{n+j} \operatorname{lcm}\left(\bar{\alpha}_{i-j}, \bar{\gamma}_{i}\right), \quad j \leq m-n
$$

and let

$$
\sigma_{j}(\bar{\alpha}, \bar{\gamma})=\frac{\pi_{j}(\bar{\alpha}, \bar{\gamma})}{\pi_{j-1}(\bar{\alpha}, \bar{\gamma})}, \quad j \leq m-n
$$

Then

$$
\sigma_{j}(\bar{\alpha}, \bar{\gamma}) \mid \sigma_{j+1}(\bar{\alpha}, \bar{\gamma}), \quad j \leq m-n-1
$$

In this section, we shall prove that the integers $f_{1}, \ldots, f_{s}$ defined by (3.20) as well as the integers $w_{1}, \ldots, w_{x}$ defined by (3.21) are both non-increasing, thus defining partitions $\mathbf{f}=\left(f_{1}, \ldots, f_{s}\right)$ and $\mathbf{w}=$ $\left(w_{1}, \ldots, w_{x}\right)$.

Let $\gamma_{i} \mid \alpha_{i}, i=1, \ldots, n$. By using definitions (4.78) and (4.79), from (3.20) we have

$$
f_{j}=\sum_{i=1}^{n+s-j+1} d\left(\operatorname{lcm}\left(\alpha_{i-s+j-1}, \gamma_{i}\right)\right)-\sum_{i=1}^{n+s-j} d\left(\operatorname{lcm}\left(\alpha_{i-s+j}, \gamma_{i}\right)\right)-1=d\left(\sigma_{s-j+1}(\alpha, \gamma)\right)-1
$$


for all $j=1, \ldots, s-1$, and

$$
f_{j+1}=\sum_{i=1}^{n+s-j} d\left(\operatorname{lcm}\left(\alpha_{i-s+j}, \gamma_{i}\right)\right)-\sum_{i=1}^{n+s-j-1} d\left(\operatorname{lcm}\left(\alpha_{i-s+j+1}, \gamma_{i}\right)\right)-1=d\left(\sigma_{s-j}(\alpha, \gamma)\right)-1
$$

for all $j=1, \ldots, s-1$. Thus, by Lemma 4.1 , we get $f_{j} \geq f_{j+1}, j=1, \ldots, s-1$, as wanted.

Let the condition ( iii) from Theorem 3.1 be valid, i.e., let (3.22) be satisfied.

From (3.21), we have

$$
\sum_{i=j}^{x} w_{i}=\max _{k=0, \ldots, y-s}\left[\sum_{i=1}^{n+s} d\left(\operatorname{lcm}\left(\alpha_{i-s-j-k+1}, \gamma_{i}\right)\right)-\sum_{i=k+1}^{y-s} \bar{r}_{i}-\sum_{i=1}^{n+s} d\left(\gamma_{i}\right)\right]
$$

for all $j=1, \ldots, x$. Let us denote by

$$
F(j, k):=\sum_{i=1}^{n+s} d\left(\operatorname{lcm}\left(\alpha_{i-s-j-k+1}, \gamma_{i}\right)\right)-\sum_{i=k+1}^{y-s} \bar{r}_{i}-\sum_{i=1}^{n+s} d\left(\gamma_{i}\right)
$$

for all $j=1, \ldots, x$, and $k=0, \ldots, y-s$.

For any $j=1, \ldots, x$, let $k_{j} \in\{0, \ldots, y-s\}$ be such that

$$
\sum_{i=j}^{x} w_{i}=\sum_{i=1}^{n+s} d\left(\operatorname{lcm}\left(\alpha_{i-s-j-k_{j}+1}, \gamma_{i}\right)\right)-\sum_{i=k_{j}+1}^{y-s} \bar{r}_{i}-\sum_{i=1}^{n+s} d\left(\gamma_{i}\right)=F\left(j, k_{j}\right),
$$

i.e., for every $j=1, \ldots, x$, we have

$$
F\left(j, k_{j}\right) \geq F(j, k), \quad k=0, \ldots, y-s .
$$

For every $j=1, \ldots, x-1$, we have

$$
\begin{aligned}
w_{j} & =\sum_{i=j}^{x} w_{i}-\sum_{i=j+1}^{x} w_{i}=F\left(j, k_{j}\right)-F\left(j+1, k_{j+1}\right) \\
& \geq F\left(j, k_{j+1}\right)-F\left(j+1, k_{j+1}\right)=\sum_{i=1}^{n+s} d\left(\operatorname{lcm}\left(\alpha_{i-s-j+1-k_{j+1}}, \gamma_{i}\right)-\sum_{i=1}^{n+s} d\left(\operatorname{lcm}\left(\alpha_{i-s-j-k_{j+1}}, \gamma_{i}\right)\right.\right. \\
& =d\left(\pi_{-s-j+1-k_{j+1}}(\gamma, \alpha)\right)-d\left(\pi_{-s-j-k_{j+1}}(\gamma, \alpha)\right)=d\left(\sigma_{-s-j+1-k_{j+1}}(\gamma, \alpha)\right) .
\end{aligned}
$$

Also, for every $j=1, \ldots, x-2$, we have

$$
\begin{aligned}
w_{j+1} & =\sum_{i=j+1}^{x} w_{i}-\sum_{i=j+2}^{x} w_{i}=F\left(j+1, k_{j+1}\right)-F\left(j+2, k_{j+2}\right) \\
& \leq F\left(j+1, k_{j+1}\right)-F\left(j+2, k_{j+1}\right) \\
& =\sum_{i=1}^{n+s} d\left(\operatorname{lcm}\left(\alpha_{i-s-j-k_{j+1}}, \gamma_{i}\right)-\sum_{i=1}^{n+s} d\left(\operatorname{lcm}\left(\alpha_{i-s-j-1-k_{j+1}}, \gamma_{i}\right)\right.\right. \\
& =d\left(\sigma_{-s-j-k_{j+1}}(\gamma, \alpha)\right) .
\end{aligned}
$$


Moreover, by condition (3.22) for $k=k_{x}$, we have

$$
\begin{aligned}
w_{x} & =\sum_{i=1}^{n+s} d\left(\operatorname{lcm}\left(\alpha_{i-s-x-k_{x}+1}, \gamma_{i}\right)\right)-\sum_{i=k_{x}+1}^{y-s} \bar{r}_{i}-\sum_{i=1}^{n+s} d\left(\gamma_{i}\right) \\
& \leq \sum_{i=1}^{n+s} d\left(\operatorname{lcm}\left(\alpha_{i-s-x-k_{x}+1}, \gamma_{i}\right)\right)-\sum_{i=1}^{n+s} d\left(\operatorname{lcm}\left(\alpha_{i-s-x-k_{x}}, \gamma_{i}\right)\right) \\
& =d\left(\sigma_{-s-x+1-k_{x}}(\gamma, \alpha)\right)
\end{aligned}
$$

Therefore, for all $j=1, \ldots, x-1$, we have

$$
w_{j+1} \leq d\left(\sigma_{-s-j-k_{j+1}}(\gamma, \alpha)\right)
$$

By applying Lemma 4.1, (4.83) and (4.84) give $w_{j} \geq w_{j+1}$ for all $j=1, \ldots, x-1$, as wanted.

Acknowledgements. We thank the referee for improvements of the manuscript.

\section{REFERENCES}

[1] I. Baragaña. Interlacing inequalities for regular pencils. Linear Algebra Appl., 121:521-535, 1989.

[2] I. Baragaña and I. Zaballa. Column completion of a pair of matrices. Linear Multilinear Algebra, 27:243-273, 1990.

[3] G. Basile and G. Marro. Controlled and Conditional Invariants in Linear Systems Theory. Prentice-Hall, Englewood Cliffs, NJ, 1992.

[4] I. Cabral and F.C. Silva. Similarity invariants of completions of submatrices. Linear Algebra Appl., 169:151-161, 1992.

[5] M. Dodig and M. Stošić. Similarity class of a matrix with prescribed submatrix. Linear Multilinear Algebra, 57:217-245, 2009.

[6] M. Dodig. Matrix pencils completion problems II. Linear Algebra Appl., 429:633-648, 2008.

[7] M. Dodig. Descriptor systems under feedback and output injection. In: C. André, M.A. Bastos, A.Yu. Karlovich, B. Silbermann, and I. Zaballa (editors), Operator Theory, Operator Algebras, and Matrix Theory, 141-166, 2018.

[8] M. Dodig and M. Stošić. The general matrix pencil completion problem - a minimal case. SIAM J. Matrix Anal. Appl., 40(1):347-369, 2019.

[9] M. Dodig. Completion of quasi-regular matrix pencils. Linear Algebra Appl., 501:198-241, 2016.

[10] M. Dodig. Minimal completion problem for quasi-regular matrix pencils. Linear Algebra Appl., 525:84-104, 2017.

[11] M. Dodig and M. Stošić. More on the properties of the generalized majorization. Preprint, arXiv:1905.08053, 2019.

[12] M. Dodig and M. Stošić. Combinatorics of polynomial chains. Linear Algebra Appl. 589:130-157, 2020.

[13] M. Dodig and M. Stošić. The rank distance problem for pairs of matrices and a completion of quasi-regular matrix pencils. Linear Algebra Appl., 457:313-347, 2014.

[14] M. Dodig. Explicit solution of the row completion problem for matrix pencils. Linear Algebra Appl., 432:1299-1309, 2010.

[15] M. Dodig. Completion up to a matrix pencil with column minimal indices as the only nontrivial Kronecker invariants. Linear Algebra Appl., 438:3155-3173, 2013.

[16] M. Dodig and M. Stošić. Combinatorics of column minimal indices and matrix pencil completion problems. SIAM J. Matrix Anal. Appl., 31:2318-2346, 2010.

[17] M. Dodig and M. Stošić. On convexity of polynomial paths and generalized majorizations. Electron. J. Combin., 17(1):R61, 2010.

[18] M. Dodig and M. Stošić. On properties of the generalized majorization. Electron. J. Linear Algebra, 26:471-509, 2013.

[19] M. Dodig. Matrix pencils completion problems. Linear Algebra Appl., 428(1):259-304, 2008.

[20] J.C. Doyle. Guaranteed margins for LQG regulators. IEEE Trans. Automat. Contr., 23:756-757, 1978.

[21] I. Gohberg, M.A. Kaashoek, and F. van Schagen. Eigenvalues of completions of submatrices. Linear Multilinear Algebra, 25:55-70, 1989

[22] Y. Han. Subrepresentations of Kronecker representations. Linear Algebra Appl., 402:150-164, 2005.

[23] M. Heymann. Controllability indices and feedback simulation. SIAM J. Control Optim., 14:769-789, 1976. 
[24] G. de Oliveira. Matrices with prescribed characteristic polynomial and a prescribed submatrix III. Monatsh. Math., 75:441-446, 1971.

[25] G. de Oliveira. Matrices with prescribed entries and eigenvalues II. SIAM J. Appl. Math., 24:414-417, 1983.

[26] J. Loiseau, S. Mondié, I. Zaballa, and P. Zagalak. Assigning the Kronecker invariants of a matrix pencil by row or column completion. Linear Algebra Appl., 278:327-336, 1998.

[27] E.M. Sá. Imbedding conditions for $\lambda$-matrices. Linear Algebra Appl., 24:33-50, 1979.

[28] C. Szanto. Submodules of Kronecker modules via extension monoid products. J. Pure Appl. Algebra, 222:3360-3378, 2018.

[29] R.C. Thompson. Interlacing inequalities for invariant factors. Linear Algebra Appl., 24:1-31, 1979.

[30] H.K. Wimmer. Existenzsatze in Theorie der Matrizen und Lineare Kontrolltheorie, Monatsh. Math., 78:256-263, 1974.

[31] I. Zaballa. Matrices with prescribed rows and invariant factors. Linear Algebra Appl., 87:113-146, 1987.

[32] I. Zaballa. Interlacing inequalities and control theory. Linear Algebra Appl., 101:9-31, 1988. 